\title{
Coulomb blockade of spin-dependent shuttling
}

\author{
Hee Chul Park ${ }^{1}$, Anatoli M. Kadigrobov ${ }^{2,3}$, Robert I. Shekhter ${ }^{2}$, and M. Jonson ${ }^{2,4,5}$ \\ ${ }^{1}$ School of Computational Science, Korea Institute for Advanced Study, Seoul 130-722, Korea \\ E-mail: hcpark@kias.re.kr \\ ${ }^{2}$ Department of Physics, University of Gothenburg, Göteborg SE-412 96, Sweden \\ ${ }^{3}$ Theoretische Physik III, Ruhr-Universität Bochum, Bochum D-44801, Germany \\ ${ }^{4}$ SUPA, Institute of Photonics and Quantum Sciences, Heriot-Watt University, Edinburgh EH14 4AS, Scotland, UK \\ ${ }^{5}$ Department of Physics, Division of Quantum Phases and Devices, Konkuk University, Seoul 143-701, Korea
}

Received July 9, 2013

\begin{abstract}
We show that nanomechanical shuttling of single electrons may enable qualitatively new functionality if spinpolarized electrons are injected into a nanoelectromechanical single-electron tunneling (NEM-SET) device. This is due to the combined effects of spin-dependent electron tunneling and Coulomb blockade of tunneling, which are phenomena that occur in certain magnetic NEM-SET devices. Two effects are predicted to occur in such structures. The first is a reentrant shuttle instability, by which we mean the sequential appearance, disappearance and again the appearance of a shuttle instability as the driving voltage is increased (or the mechanical dissipation is diminished). The second effect is an enhanced spin polarization of the nanomechanically assisted current flow.
\end{abstract}

PACS: $81.07 .0 j$ Nanoelectromechanical systems (NEMS);

72.25.-b Spin-polarized transport;

73.23.Hk Coulomb blockade; single-electron tunneling.

Keywords: nanoelectromechanical systems, Coulomb blockade, spin-polarized transport, shuttle systems.

\section{Introduction}

Large Coulomb forces, which accompany the tunneling of single electrons between nanometer-sized conductors, naturally result in significant mechanical deformations of single-electron-tunneling (SET) nanodevices leading to an efficient coupling between electronic and mechanical degrees of freedom. The nanoelectromechanics (NEM) originating from such a coupling is presently one of the most quickly developing directions of nanophysics. Apart from a number of fundamentally new phenomena (such as spintro-mechanics [1], mechanically assisted macroscopic quantum interference [2-5], mechanical probing of Kondo ordering [6,7], etc.) this direction also offers a large variety of potential applications (such as the realization of highly sensitive sensors, mechanically assisted cooling and thermoelectric nanodevices [1-14]). One of the prototype nanoelectromechanical systems (NEMS) of this type, suggested in $[15,16]$, is the electron "shuttle". In this NEM-SET device electric charge is accumulated on a movable dot as the result of the flow of an electrical current. The shuttle phenomenon does not involve the electronic spin degree of freedom as an active "player" in the NEM. Additional func- tionality evidently should occur if such a decoupling between NEM and spin degrees of freedom is removed.

This situation is indeed realized if a spin-polarized current is injected into the shuttle device, which is the case if one of the leads (or both) is made of magnetic material. The phenomenon of spin-dependent tunneling, originating from the spin dependence of the electron density of states in ferromagnets, means that accumulation of both electronic charge and electronic spin on the movable "shuttle" will be affected, hence potentially modifying qualitatively the mechanics of such a current carrying device.

In this paper we will show that a simple modification of the standard shuttle device, achieved by replacing one of the non-magnetic metallic leads by a ferromagnetic metal qualitatively affects the shuttle operation of the device. Two new phenomena will be shown to occur as result of the interplay between spin-dependent tunneling and the Coulomb blockade of single-electron tunneling. Those are: 1) a reentrance of the shuttle instability, i.e., the suppression of the shuttle instability in a certain domain of driving voltages and levels of mechanical dissipation, and 2) shuttle-induced enhancement of the spin polarization of mechanically transported electron currents. 


\section{Formulation of the problem}

The system we are going to consider is presented in Fig. 1. A single-electron tunneling device is formed by two metal leads, one of which (the right one) is a ferromagnetic metal with electronic states split with respect to spin by an internal exchange interaction. The central island (a "quantum dot"), which is responsible for the Coulomb blockade of electron tunneling to and from the dot, in our case has the ability to oscillate between the two electrodes. On the one hand, these oscillations are driven by an electrical force, which is due to the electric charge accumulated on the dot as a result of the tunneling injection of electrons from the leads, and, on the other hand, the oscillations exponentially affect the probability for the same tunneling injection. In Ref. 15 this interplay was shown to result in the onset of mechanical vibrations of the dot if the driving voltage exceeds a certain critical value set by the mechanical dissipation. For the following discussion it is important that there is a finite Zeeman splitting of the electronic level on the dot. This can be achieved by applying an external magnetic field as indicated in Fig. 1. The spin of the electrons controls the nanomechanics of the device by affecting the charge accumulation on the dot in two ways: firstly through the Zeeman splitting if the (single) electron energy level on the dot and secondly through the spin dependence of the probability for tunneling to the right electrode. To be concrete we will suppose that without any driving voltage $(V=0)$ the two split levels on the dot are situated above the

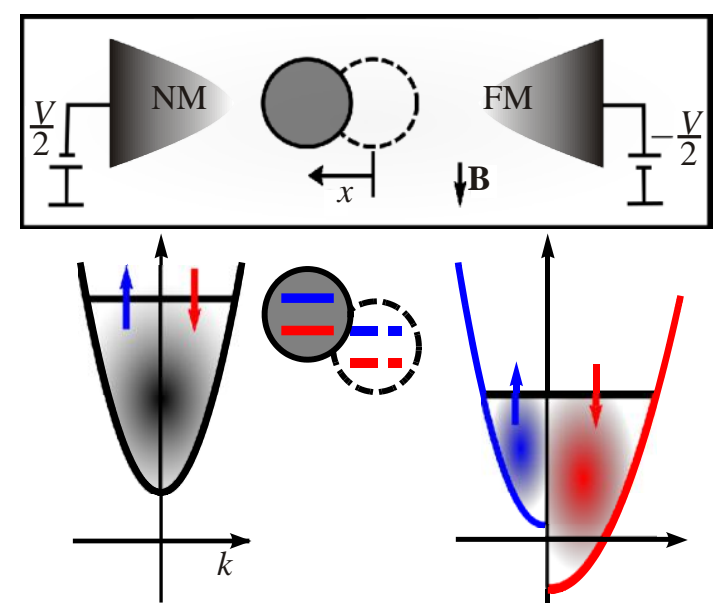

Fig 1. (Color online) Schematic figure of the studied nanoelectromechanical single-electron tunneling (NEM-SET) device with corresponding energy diagrams for the left (normal metal) source electrode, the central movable island (the "quantum dot") and the right (ferromagnetic) drain electrode. The Zeeman splitting of the single-electron energy level on the quantum dot, $\Delta \varepsilon=\varepsilon_{\uparrow}-\varepsilon_{\downarrow}$, is caused by an external magnetic field $\mathbf{B}$ pointing down as shown. Dashed lines indicate the equilibrium position of the dot and its split energy level in the absence of any bias voltage $V$. Due to the electric field associated with an applied bias voltage the split levels move up in energy as the dot is displaced towards the left electrode. common chemical potential in the leads, such that at low temperatures even the bottom spin-down level is not populated. Switching on a finite driving voltage induces additional potentials on the leads $V_{L(R)}: V_{L}=-V_{R}=V / 2$. As a result, only the left electrode can serve as a source of electrons that can tunnel onto the dot, while the right electrode serves only as a voltage-independent drain of tunneling electrons.

Switching on tunneling opens the possibility to occupy both the spin-up and the spin-down electronic levels on the dot. The probability $P_{\sigma}$ of occupying the state with spin $\sigma$ obeys a constraint $\left(P_{0}\right.$ is the probability to have an unoccupied dot) since

$$
P_{0}+P_{\uparrow}+P_{\downarrow}=1 .
$$

Equation (1) tells us that the dot can not be doubly occupied in the deep Coulomb blockade regime. In the limit of weak tunneling the kinetics of the charge/spin accumulation on the dot obeys a kinetic equation that can be expressed in terms of the tunneling rates $\gamma_{s \sigma}$, where

$$
\gamma_{s \sigma}=\gamma_{s \sigma}(0) \mathrm{e}^{ \pm x / \lambda}, \quad s=L, R
$$

Here the upper sign (+) in the exponent corresponds to the subscript $s=R$ in $\gamma_{s \sigma}$ and the lower sign (-) corresponds to $s=L$. Note that the tunneling rates are exponentially sensitive to any displacement $x$ of the dot from its (zerovoltage) equilibrium position in the middle of the contact (see Fig. 1). Furthermore,

$$
\gamma_{s \sigma}(0)=(2 \pi / \hbar) D_{0} g_{s \sigma}
$$

where $D_{0}$ is transparency of the barriers between the dot and either lead and $g_{S \sigma}$ is the density of electronic states in the left and right leads. In the ferromagnetic right lead $g_{R \sigma}$ is spin-dependent as a result of the exchange interaction induced Zeeman level splitting, whereas in the left normalmetal lead $g_{L \uparrow}=g_{L \downarrow}$ and therefore $\gamma_{L \uparrow}=\gamma_{L \downarrow}=\gamma_{L}$, independent of spin.

The kinetic equation for $P_{\sigma}$ can be derived as in Ref. 17 . Taking into account the constraint (1) only the two quantities $P_{\uparrow}$ and $P_{\downarrow}$ are independent variables. Their time development is governed by the equation

$$
\frac{d P_{\sigma}}{d t}=I_{L \sigma}-I_{R \sigma} l e, \quad \sigma=\uparrow, \downarrow
$$

where the (time-dependent) spin currents from the left lead to the dot, $I_{L \sigma}$, and from the dot to the right lead, $I_{R \sigma}$, can be calculated as

$$
\begin{aligned}
& I_{L \sigma}=e \gamma_{L}\left\{f_{L \sigma}-P_{\sigma}-f_{L \sigma} P_{\bar{\sigma}}\right\}, \\
& I_{R \sigma}=e \gamma_{R \sigma} P_{\sigma} .
\end{aligned}
$$

In Eq. (5) $\bar{\sigma}$ and $\sigma$ denote antiparallel spins and $f_{L \sigma}$ is the Fermi-Dirac function

$$
f_{L \sigma}=\frac{1}{1+\mathrm{e}^{\beta\left(\varepsilon_{\sigma}-e V_{L}\right)}},
$$


which gives the equilibrium occupation probability for the electronic state that can tunnel elastically into the dot's energy level $\varepsilon_{\sigma}$ (which have kinetic energy $\varepsilon_{\sigma}-e V_{L}$ ).

The average charge $\langle Q\rangle$ accumulated on the dot, which can be expressed in terms of the probabilities $P_{\sigma}$ as

$$
\langle Q\rangle=e \sum_{\sigma=\uparrow, \downarrow} P_{\sigma},
$$

couples to the electric field, which we approximate by the ratio $V / L$ of the driving voltage $V$ and the distance $L$ between the electrodes where the potential drops. The result is an electric force acting on the vibrating dot in accordance with the classical Newton's equation [17]

$$
m \ddot{x}+m \gamma \dot{x}+m \omega^{2} x=\langle Q\rangle V / L .
$$

Here $x$ is the displacement of the dot and $m$ is its mass while $\omega$ is the angular oscillation frequency and $\gamma$ the damping rate of the uncharged dot.

The charge and spin carried by the dot can be naturally expressed in terms of the quantities $P_{ \pm}$, where

$$
P_{ \pm}=P_{\uparrow} \pm P_{\downarrow}
$$

Using Eqs. (4) and (5) it is straightforward to derive two equations for $P_{ \pm}$. These can be economically written as

$$
\begin{aligned}
\frac{d P_{ \pm}}{d t} & =-\gamma_{L}\left\{\left(1+\rho_{+} \pm f_{L+}\right) P_{ \pm}+\right. \\
& \left.+\left(\rho_{-} \mp f_{L-}\right) P_{\mp}-f_{L_{ \pm}}\right\},
\end{aligned}
$$

where $f_{L_{ \pm}}=f_{L+} \pm f_{L_{-}}$and the spin and charge asymmetry parameters $\rho_{ \pm}$are defined as

$$
\rho_{ \pm}=\frac{1}{2} \rho_{\uparrow} \pm \rho_{\downarrow}, \quad \rho_{\sigma}=\gamma_{R \sigma} / \gamma_{L}
$$

Since the effects we are going to discuss have to do with the spin-splitting of the electronic level $\varepsilon_{\sigma}$ on the dot, the magnitude $\Delta \varepsilon$ of this splitting, where

$$
\Delta \varepsilon=\varepsilon_{\uparrow}-\varepsilon_{\downarrow}
$$

gives a proper scale for the voltage $V$ applied to the system. By normalizing the electric force to $m \omega^{2} \lambda$, which gives the scale for the elastic force, we can define a dimensionless electric force $F$ acting on the shuttle and express it as

$$
F \equiv \frac{\langle Q\rangle V / L}{m \omega^{2} \lambda}=\frac{\Omega^{2}}{\omega^{2}} \frac{e V}{2 \Delta \varepsilon} P_{+},
$$

where

$$
\Omega=\sqrt{\frac{2 \Delta \varepsilon}{m \lambda L}} .
$$

Finally, the time-dependent electrical charge current $I_{L}(V, t)$ from the left lead to the dot and the charge cur- rent $I_{R}(V, t)$ from the dot to the right lead can be calculated by solving Eq. (4) for $P_{\sigma}$ [or equivalently Eq. (10) for $\left.P_{ \pm}\right]$together with Eq. (5) and then sum the "local" spin currents $I_{L \sigma}=I_{L \sigma}(V, t)$ and $I_{R \sigma}=I_{R \sigma}(V, t)$ defined by Eq. (5) over both spin directions $\sigma=\uparrow, \downarrow$ to get

$$
\begin{gathered}
I_{L}(V, t)=e \gamma_{L}\left\{f_{L \uparrow}\left(1-P_{\downarrow}\right)+f_{L \downarrow}\left(1-P_{\uparrow}\right)-\left(P_{\uparrow}+P_{\downarrow}\right)\right\}, \\
I_{R}(V, t)=-e\left\{\gamma_{R \uparrow} P_{\uparrow}+\gamma_{R \downarrow} P_{\downarrow}\right\} .
\end{gathered}
$$

The local spin currents $I_{L \sigma}(V, t)$ and $I_{R \sigma}(V, t)$ and the local charge currents $I_{L}(V, t)$ and $I_{R}(V, t)$ are not the same at every instant of time. However, it is possible to show that they oscillate in time with a relative phase shift corresponding to half a shuttle oscillation period $\mathcal{T}=2 \pi / \omega$. The time averaged values of these local currents, defined as

$$
I(V)=\frac{1}{\mathcal{T}} \int_{t}^{t+\mathcal{T}} I_{L(R)}(V, t) d t
$$

and

$$
I_{\sigma}(V)=\frac{1}{\mathcal{T}} \int_{t}^{t+\mathcal{T}} I_{L(R) \sigma}(V, t) d t
$$

respectively, are such that on average there is no accumulation of charge or spin on the dot.

\section{NEM assisted current in the absence of shuttle vibrations}

If the displacement of the dot is small compared to the tunneling length $\lambda$ one can find the electrical force $F$ defined in Eq. (13) by perturbation theory in terms of the small parameter $x / \lambda$. It is important that this force contributes to the equation of mechanical motion (8) with a term proportional to the velocity $\dot{x}$. This means that the electromechanical coupling renormalizes the damping of the mechanical vibration in such a way that it is diminished. Since the electric force is directly proportional to the driving voltage there is a certain critical voltage $V=V_{\text {th }}$ for which the initial mechanical damping is totally compensated. For larger voltages, $V>V_{\mathrm{th}}$, energy is pumped into the mechanical subsystem as a result of the to coupling to electrons, the source of the energy being the electric power $I(V) V$ supplied by the battery. As a result of the pumping the amplitude of any small random displacement of the dot is amplified, which corresponds to an instability against the formation of pronounced shuttle vibrations [17]. The condition for such an instability to occur is that the net work done by the electric force over one vibration period exceeds the energy lost to the environment through damping (mechanical friction). In this section we will consider the limit of small voltages, $V<V_{\text {th }}$, when mechanical vibrations do not develop and hence the position of the dot is 
independent of time. However, there is a nanomechanical effect on the current even in this limit. This is because the equilibrium position of the dot changes with voltage since the elastic restoring force of the oscillator is able to balance the electrostatic force $F$ due to the accumulated electric charge on the dot.

Using the time-independent solution of the equation of motion one obtains the shift of the equilibrium dot position $x_{0}$ (provided that $x_{0}$ is smaller than the tunneling length $\lambda)$ as

$$
\frac{x_{0}}{\lambda}=\frac{\Omega^{2}}{\omega^{2}} \frac{e V}{2 \Delta \varepsilon}\left(P_{\uparrow}^{(0)}+P_{\downarrow}^{(0)}\right) .
$$

Here $P_{\sigma}^{(0)}$ is the time-independent solution of Eq. (4), i.e.,

$$
P_{\sigma}^{(0)}=\frac{\left(1+\rho_{\bar{\sigma} 0}-f_{L \bar{\sigma}}\right) f_{L \sigma}}{\left(1+\rho_{\uparrow 0}\right)\left(1+\rho_{\downarrow 0}\right)-f_{L \uparrow} f_{L \downarrow}},
$$

where $\rho_{\sigma 0}$, the asymmetry parameter at the equilibrium position, is defined as

$$
\rho_{\sigma 0}=\gamma_{R \sigma 0} / \gamma_{L 0}
$$

One readily finds that the current $I_{0}(V)$ through a nonoscillating NEM-SET device is given by the expression

$$
I_{0}(V)=e J_{0}(V)\left[1+\frac{x_{0}}{\lambda} \frac{\gamma_{L}(0)-J_{0}(V)}{\gamma_{L}(0)}\right]
$$

The current-voltage characteristics in this nonvibrating regime is mainly given by the function $J_{0}(V)$, which is found to have the form

$$
J_{0}(V)=\gamma_{L}(0)\left\{\begin{array}{cl}
\frac{1}{1+1 / \rho_{\downarrow}(0)} f_{L \downarrow}, & e V<\varepsilon_{\uparrow}, \\
\frac{1}{1+1 / \rho_{\downarrow}(0)+1 / \rho_{\downarrow}(0)}, & e V>\varepsilon_{\uparrow} .
\end{array}\right.
$$

The result (22) for $J_{0}(V)$ allows an interesting observation to be made, viz. that if tunneling to the ferromagnet from the upper level $\varepsilon_{\uparrow}$ is significantly suppressed compared with tunneling from the lower level $\varepsilon_{\downarrow}$ then the differential conductance $d I(V) / d V$ becomes negative when the voltage $V$ is increased above $\varepsilon_{\uparrow} / e$.

There is a clear physical explanation in terms of the Coulomb blockade phenomenon and spin-dependent tunneling for this reduction of the current as the Fermi level of the left lead crosses the position of the upper energy level on the dot $[18,19]$. We note that even though the Coulomb blockade only allows one electron at a time to reside on the dot there might be more than one channel for tunneling from the source electrode (left lead) to the dot. Indeed, only if the Fermi level of the source is below the upper (spin-up) level $\varepsilon_{\uparrow}$ on the dot is there a single channel for tunneling. This is the channel where spin-down electron tunnels to the spin-down (lower) dot energy level $\varepsilon_{\downarrow}$. How- ever, if the Fermi level of the source electrode is higher than $\varepsilon_{\uparrow}$ then there is an additional channel corresponding to the tunneling of spin-up electrons from source to dot.

Next we would like to exploit the fact that the rate of further tunneling of an electron from the dot to the drain electrode (right lead) depends on the spin of the tunneling electron. Let us assume that tunneling from the upper (spin-up) level is suppressed compared with tunneling from the lower (spin-down) level because of a difference in the spin densities of final states in the ferromagnetic drain electrode. It this case we have a situation where the "upper channel", through which spin-up electrons tunnel, is "more resistive" than the "lower channel" for spin-down electrons. It follows that if the driving voltage is small enough for the Fermi level in the source electrode to be lower than the upper dot level, then all electrons flow from source to drain through the less resistive "lower channel". However, if the voltage is increased so that the source Fermi level becomes higher than the upper dot level, then some electrons will tunnel through the more resistive "upper channel" (and will have to wait longer on the dot - while blocking a second electron to tunnel from the source before it tunnels onwards to the drain). As a result an increase of voltage that makes the Fermi energy in the source electrode cross the upper dot level results in a reduction of the electrical current through the device. We stress again that this happens only when tunneling through the upper (spin-up) dot level is discriminated with respect to tunneling through the lower (spin-down) level.

\section{Reentrant shuttling of spin-polarized electrons}

In the previous section we found that the current through a stationary dot is reduced at voltages when singleelectron tunneling through two spin channels rather than only one is allowed, i.e., when tunneling may occur via both the spin-split levels $\varepsilon_{\downarrow}$ and $\varepsilon_{\uparrow}$ on the dot. If the rate of mechanical dissipation could be reduced to make those voltages large enough to produce a shuttle instability the consequences of a drop in the current should be even more dramatic. In fact, a reduced current at a given voltage translates into less power supplied to the electrons by the battery and hence it is possible that the electrons may not be able to transfer enough power to the mechanical subsystem to overcome the damping. This would mean that by increasing the driving voltage within the shuttling regime the shuttle motion could become damped at a certain voltage and the nonvibrating regime restored. However, by further increasing the voltage the power supplied to the mechanical subsystem via the electrons may again be large enough to for a shuttle instability to occur.

Such a reentrance of the shuttling regime as a function of increasing voltage can be demonstrated by solving Eqs. (10), (15), and (16) for arbitrary voltages and dissipation rates. As mentioned above the shuttle instability occurs at a cer- 
tain threshold value of the driving voltage, which depends on the rate of mechanical dissipation $\gamma$. A numerical analysis of Eqs. (4) [or (10)] and (8) allows us to identify the domains (in the voltage-dissipation plane) of nanoelectromechanical stability of the shuttle device where shuttle vibrations do not occur and the regions of shuttle instability where they do. The corresponding shuttle-instability "phase diagram" is shown in Fig. 2, which clearly demonstrates the reentrant nature of the spin-controlled nanoelectromechanical shuttle instability (see also Ref. 20 where the phenomenon of reentrant shuttling was predicted for unpolarized electrons in the regime of strong electronvibron interaction).

Figure 2 illustrates, as mentioned above, the effect of Coulomb blockade of spin-dependent tunneling, which may result in a sudden drop of the electrical power supplied to the system and a transition from a shuttling regime to a stationary regime as the driving voltage is increased. To further illustrate this effect we have performed a numerical analysis, which shows that the shuttle instability develops into pro-

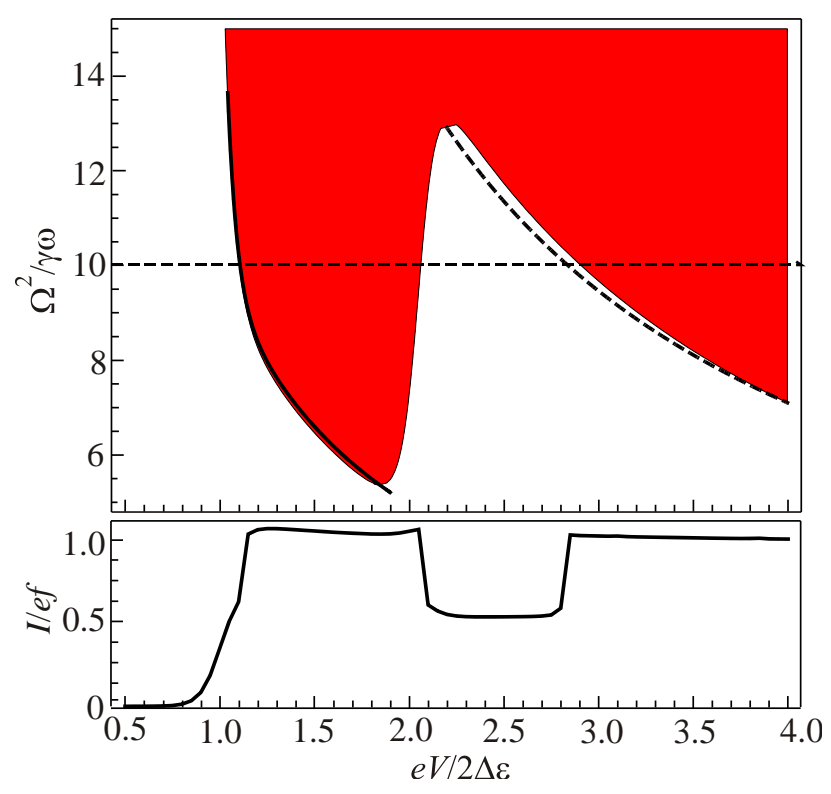

Fig. 2. (Color online) Diagram indicating regions of mechanical stability (white) and instability (red/gray) of the NEM-SET device shown in Fig. 1. The inverse mechanical dissipation rate $1 / \gamma$ is plotted in units of $\omega / \Omega^{2}$ along the vertical axis and the driving voltage in units of $2 \Delta \varepsilon / e$ along the horizontal axis. The parameters used were $\varepsilon_{\downarrow}=\Delta \varepsilon, \quad \varepsilon_{\uparrow}=2 \Delta \varepsilon, \quad \rho \uparrow(0)=0.07, \quad \rho_{\downarrow}(0)=0.13$, $k_{B} T=0.05 \Delta \varepsilon$, and $\Omega=0.1 \omega$. The solid and dashed black curves are the analytical results for the border lines between stable and instable regions given by Eqs. (A.13) and (A.14) of the Appendix. As can be seen they closely match the results of the full numerical calculation described in the text. The lower part of the figure shows the current along the horizontal dashed line in the upper part and illustrates the variation of the current that is the result of the entrance, leaving and reentrance into the unstable domain of shuttle vibrations.

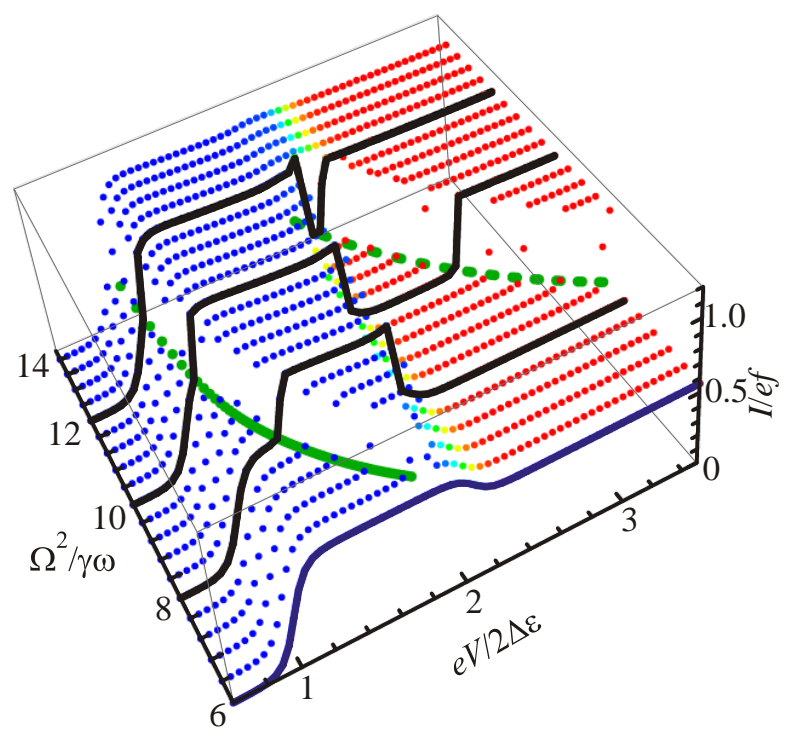

Fig. 3. (Color online) Current-voltage characteristics for the NEM-SET device shown in Fig. 1 calculated for different values of the normalized inverse dissipation rate $\Omega^{2} /(\gamma \omega)$ using the same parameters as in Fig. 2. When the threshold voltage for the shuttle instability is reached (solid green curve), approximately one (spin-down) electron is on average mechanically transported between the source and drain electrodes for each period of the shuttle vibrations, so that $I \simeq$ ef where $f=\omega / 2 \pi$. As the voltage is further increased the channel for tunneling of spin-up electrons is eventually opened, which leads to a reduced current as the vibrations are damped out (see text). At still higher voltages (dotted green curve) the shuttling domain is reentered and the current increases again.

nounced shuttle vibrations with a voltage-dependent amplitude leading to a time dependent current. A set of plots of the (charge) current, averaged according to Eq. (16), is shown as a function of the driving voltage in Fig. 3.

\section{Shuttling of spin-polarized electrons}

In Sec. 3 we showed that the value of the driving voltage $V$ controls whether electrons in the source electrode can be injected only into the lower spin-split energy level on the quantum dot or if injection into the upper level is also energetically possible. The driving voltage therefore affects the spin polarization of the electrical current that flows through the device, which we expect to be reduced when the voltage reaches a threshold value that makes the Fermi level in the left (source) electrode cross the upper spin-split level on the dot. One can easily convince oneself that the closer the dot is situated to the left electrode the higher voltage is needed to switch on the second (spin-up) channel for electron tunneling. This fact makes the whole picture sensitive to the amplitude of the shuttle vibrations. In general, the onset of shuttle vibrations and their increasing amplitude with increased voltage $V$ tends to prevent a 
significant spin polarization of the current. This is because the increasing oscillation amplitude that accompanies an increasing voltage moves the left turning point of the vibrating dot closer to the source electrode and hence decreases the probability for spin-up electrons to tunnel into the upper level on the dot, as indicated in Fig. 1.

To illustrate this effect we have in Fig. 4 plotted the normalized spin-polarized current $I_{P}$, defined as

$$
I_{P}=\frac{I_{\downarrow}-I_{\uparrow}}{I_{\downarrow}+I_{\uparrow}},
$$

where the spin currents $I_{\uparrow}$ and $I_{\downarrow}$ are averaged according to Eq. (17), as a function of driving voltage for different values of the mechanical dissipation rate. Indeed, the figure shows that for such a low dissipation rate that shuttling occurs, a high degree of spin polarization survives for higher voltages. We therefore conclude that mechanical transportation enhances the spin polarization of the electrical current through a magnetic shuttle device.

In the absence of shuttle vibrations the nanomechanical effect on the spin-polarized current can be calculated analytically with the result

$$
I_{P 0} \simeq \frac{1+\rho \uparrow 0}{\left.1+2 \frac{\Omega^{2}}{\omega^{2}} \frac{e V}{\Delta \varepsilon}\right)},
$$

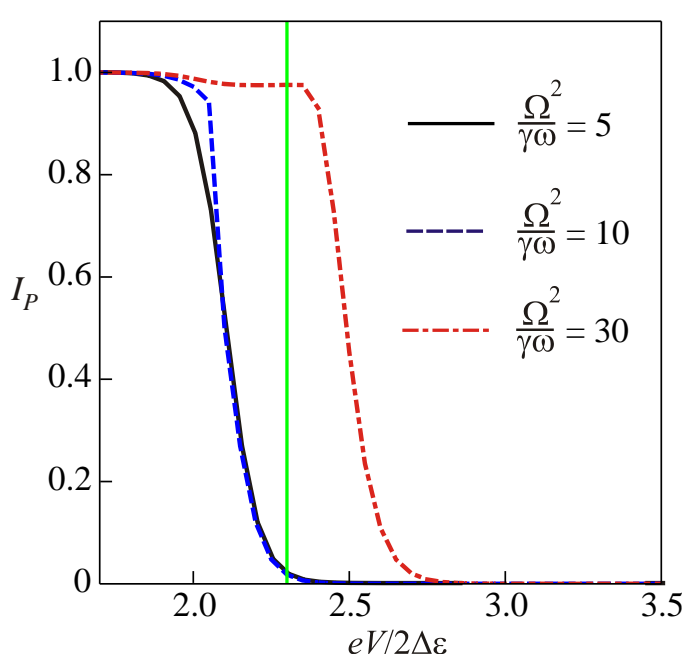

Fig. 4. (Color online) Spin polarization of the current through the NEM-SET device shown in Fig. 1 as a function of driving voltage and measured by the parameter $I_{P}=\left(I_{\downarrow}-I_{\uparrow}\right) /\left(I_{\downarrow}+I_{\uparrow}\right)$ calculated using the same parameters as in Fig. 2. Plots are shown for (from left to right) decreasing mechanical dissipation rates $\Omega^{2} /(\gamma \omega)=5.0$ (black solid line), 10.0 (blue dashed line), and 30.0 (dot-dashed red line). A vertical green line indicates where the mechanical enhancement of the spin polarization starts to become important due to the onset of shuttle vibrations.
Here $\varphi$ is a factor that controls the population of the upper level on the dot:

$$
\varphi=1+2 \exp -\beta(\varepsilon \uparrow-e V)
$$

\section{Conclusion}

In this paper we have shown that injection of spinpolarized electrons into a single-electron shuttle device drastically changes the shuttle phenomenon as such. Reentrance of the shuttle instability domain in parameter space and a mechanically enhanced spin polarization of the current are two qualitatively new effects resulting from the interplay between the Coulomb blockade of tunneling and the spin dependence of tunneling on the one hand and the effect of mechanical charge transportation on the other hand. Coulomb blockade of single electron tunneling is essential for the reentrance phenomenon and this makes spincontrolled shuttling sensitive to the possible influence of a gate potential. The periodic lifting of the Coulomb blockade as the gate voltage is varied should trigger the shuttle mechanics and give rise to pronounced shuttle vibrations. This effect complements a number of spintronic Coulomb blockade phenomena discussed in recent years for nonmechanical single-electron tunneling devices [19,21,22]. Further new functionalities can be achieved if electronic spin-flips caused either by external microwave or magnetic fields will be taken into account. A discussion of such effects is, however, outside the scope of the present paper.

Financial support from the Swedish VR and the Korean WCU program funded by MEST/NFR (R31-2008-00010057-0) is gratefully acknowledged. Computations were supported by CAC of KIAS. CHP thanks the Department of Physics, University of Gothenburg, for hospitality.

\section{Appendix}

By a linearization of Eqs. (4) and (8) in the neighborhood of a stationary state the stability of the system can be determined by using the well known Routh-Hurwitz stability criterion. Substituting the trial solution, $\tilde{x}_{0} \mathrm{e}^{\chi \tau}$, into the linearized equations yields the algebraic equation

$$
\operatorname{Det}\left(\begin{array}{ccc}
\chi+a_{\uparrow 1} & a_{\uparrow 2} & a_{\uparrow 3} \\
a_{\downarrow 2} & \chi+a_{\downarrow 1} & a_{\downarrow 3} \\
v & v & \chi^{2}+\tilde{\gamma} \lambda+1
\end{array}\right)=0,
$$

where $a_{\sigma 1}=\tilde{\gamma}_{L 0}\left(1+\rho_{\sigma 0}\right), \quad a_{\sigma 2}=\tilde{\gamma}_{L 0} f_{L \sigma}, \quad a_{\sigma 3}=2 \tilde{I}_{\sigma 0}$, and $v=-\tilde{\Omega}^{2} \tilde{V}$. Here $\tilde{\gamma}, \tilde{\gamma}_{L 0}, \chi$ and $\tilde{\Omega}$ are dimensionless variables (in units of $\omega$ ), and $\tilde{V} \equiv e V / 2 \Delta \varepsilon$.

Computation of the determinant leads to a polynomial expression in $\chi$ of the form

$$
\chi^{4}+e_{3} \chi^{3}+e_{2} \chi^{2}+e_{1} \chi+e_{0}=0,
$$


where

$$
\begin{gathered}
e_{3}=\tilde{\gamma}+a_{\uparrow 1}+a_{\downarrow 1}, \\
e_{2}=\tilde{\gamma}\left(a_{\uparrow 1}+a_{\downarrow 1}\right)+\tilde{\gamma}_{L 0}^{2} \Gamma_{D}+1, \\
e_{1}=\tilde{\gamma}_{L 0}^{2} \Gamma_{D}+\left(a_{\uparrow 1}+a_{\downarrow 1}\right)-2 \tilde{I}_{0}, \\
e_{0}=\tilde{\gamma}_{L 0}^{2} \Gamma_{D}-v a_{\uparrow 3} P_{\downarrow}^{(0)} \Gamma_{D}-v a_{\downarrow 3} P_{\uparrow}^{(0)} \Gamma_{D},
\end{gathered}
$$

and

$$
\Gamma_{D}=\left(1+\rho_{\uparrow}\right)\left(1+\rho_{\downarrow 0}\right)-f_{L \uparrow} f_{L \downarrow}
$$

The probabilities $P_{\sigma}^{(0)}$ and the current at the equilibrium position of the quantum dot can be obtained from Eqs. (4) and (8).

Evaluating the roots of a polynomial is tricky unless it is of order three or less. To reduce the order of the polynomial we assume that all the roots are purely imaginary, in which case the variables have stationary oscillating solutions. In this case Eq. (A.2) splits into two, one for the real part and one for the imaginary part. By combining these one finds a criterion for being on the border of stability that reads

$$
e_{1}^{2}-e_{3} e_{2} e_{1}+e_{3}^{2} e_{0}=0
$$

For voltages such that $e V<\varepsilon_{\uparrow}$ we use the static approximation for the probability that the dot is occupied by a spin-up electron, $\dot{P}_{\uparrow} \simeq 0$, and $f_{L \uparrow} \simeq 0$. We then get the two simplified coupled equations

$$
\dot{P}_{\downarrow}=-\tilde{\gamma}_{L}\left\{\left(1+\rho_{\downarrow}\right) P_{\downarrow}-f_{L, \downarrow}\right\}, \quad \ddot{\tilde{x}}+\tilde{\gamma} \dot{\tilde{x}}+\tilde{x}=\tilde{\Omega}^{2} \tilde{V} P_{\downarrow} .
$$

Linearization gives us the following condition for the onset of shuttling, which can be numerically calculated:

$$
\frac{\tilde{\Omega}^{2}}{\tilde{\gamma}} \geq \frac{a_{\downarrow 1}\left(a_{\downarrow 1}^{2}+1\right)}{4 \tilde{\gamma}_{L 0} \rho_{\downarrow 0} a_{\downarrow 2} V}\left[1+\sqrt{1+\frac{8 \tilde{\gamma}_{L 0} \rho_{\downarrow 0} a_{\downarrow 2}}{\left(a_{\downarrow 1}^{2}+1\right)^{2}} \tilde{\Omega}^{2} \tilde{V}}\right] .
$$

If $e V>\varepsilon \uparrow$ we assume that probability for occupation of the dot by either spin is approximately the same, $P_{-} \simeq 0$ and that $f_{L \downarrow} \simeq f_{L \downarrow} \simeq 1$. A criterion for reentering the shuttling regime can now be derived from the two coupled equations

$$
\dot{P}_{+}=-\tilde{\gamma}_{L}\left\{\left(2+\rho_{+}\right) P_{+}-f_{L,+}\right\}, \quad \ddot{\tilde{x}}+\tilde{\gamma} \dot{\tilde{x}}+\tilde{x}=\tilde{\Omega}^{2} \tilde{V} P_{+},
$$

with the result

$$
\begin{gathered}
\frac{\tilde{\Omega}^{2}}{\tilde{\gamma}} \geq \frac{b\left(b^{2}+1\right)}{4 \tilde{\gamma}_{L 0}^{2}\left(\rho_{\uparrow_{0}}+\rho_{\downarrow 0}\right) \tilde{V}} \times \\
\times\left[1+\sqrt{1+\frac{16 \tilde{\gamma}_{L 0}^{2}\left(\rho_{\uparrow 0}+\rho_{\downarrow 0}\right)}{\left(b^{2}+1\right)^{2}} \tilde{\Omega}^{2} \tilde{V}}\right],
\end{gathered}
$$

where $b=\tilde{\gamma}_{L 0}\left(2+\rho_{+0}\right)$ and which has to be evaluated numerically because of the implicit dependence on voltage.

In the limit $\tilde{\Omega} \simeq 1$ critical conditions simplifies and can be evaluated analytically Eqs. (A.10) and (A.12) simplify to, respectively,

$$
\frac{\Omega^{2}}{\gamma} \geq \frac{\left(1+\rho_{\downarrow}(0)\right)\left\{\omega^{2}+\gamma_{L}(0)^{2}\left(1+\rho_{\downarrow}(0)\right)^{2}\right\}}{2 \gamma_{L}(0) \rho_{\downarrow}(0) f_{\downarrow}(V)} \frac{2 \Delta \varepsilon}{e V}
$$

and

$$
\begin{array}{r}
\frac{\Omega^{2}}{\gamma} \geq \frac{4+\left(\rho_{\uparrow}(0)+\rho_{\downarrow}(0)\right)}{16 \gamma_{L}(0)\left(\rho_{\uparrow}(0)+\rho_{\downarrow}(0)\right)} \times \\
\times\left\{4 \omega^{2}+\gamma_{L}(0)^{2}\left[4+\left(\rho_{\uparrow}(0)+\rho_{\downarrow}(0)\right)\right]^{2}\right\} \frac{2 \Delta \varepsilon}{e V},
\end{array}
$$

which have been plotted in Fig. 2 .

1. R.I. Shekhter, A. Pulkin, and M. Jonson, Phys. Rev. B 86, 100404(R) (2012).

2. R.I. Shekhter, L.Y. Gorelik, L.I. Glazman, and M. Jonson, Phys. Rev. Lett. 97, 156801 (2006).

3. L.Y. Gorelik, A. Isacsson, Y.M. Galperin, R.I. Shekhter, and M. Jonson, Nature 411, 454 (2001).

4. A. Isacsson, L.Y. Gorelik, R.I. Shekhter, Y.M. Galperin, and M. Jonson, Phys. Rev. Lett. 89, 277002 (2002).

5. L.Y. Gorelik, R.I. Shekhter, V.M. Vinokur, D.E. Feldman, V.I. Kozub, and M. Jonson, Phys. Rev. Lett. 91, 088301 (2003).

6. M.N. Kiselev, K.A. Kikoin, L.Y. Gorelik, and R.I. Shekhter, Phys. Rev. Lett. 110, 066804 (2013).

7. M.N. Kiselev, K.A. Kikoin, R.I. Shekhter, and V.M. Vinokur, Phys. Rev. B 74, 233403 (2006).

8. Rui-Qiang Wang and Liang-Bin Hu, Phys. Lett. A 374, 4156 (2010).

9. F. Pistolesi and Rosario Fazio, Phys. Rev. Lett. 94, 036806 (2005).

10. Kang-Hun Ahn, Hee Chul Park, Jan Wiersig, and Jongbae Hong, Phys. Rev. Lett. 97, 216804 (2006).

11. Chulki Kim, Jonghoo Park, and Robert H. Blick, Phys. Rev. Lett. 105, 067204 (2010).

12. D. Fedorets, L.Y. Gorelik, R.I. Shekhter, and M. Jonson, Phys. Rev. Lett. 95, 057203 (2005).

13. Gustav Sonne, Milton E. Peña-Aza, Leonid Y. Gorelik, Robert I. Shekhter, and Mats Jonson, Phys. Rev. Lett. 104, 226802 (2010).

14. Rui-Qiang Wang, Baigeng Wang, and D.Y. Xing, Phys. Rev. Lett. 100, 117206 (2010).

15. L.Y. Gorelik, A. Isacsson, M.V. Voinova, B. Kasemo, R.I. Shekhter, and M. Jonson, Phys. Rev. Lett. 80, 4526 (1998).

16. L.M. Jonsson, L.Y. Gorelik, R.I. Shekhter, and M. Jonson, Nano Lett. 5, 1165 (2005).

17. R.I. Shekhter, L.Y. Gorelik, I.V. Krive, M.N. Kiselev, A.V. Parafilo, and M. Jonson, Nanoelectromechanical Systems 1, 1 (2013). 
18. D. Radi, A.M. Kadigrobov, L.Y. Gorelik, R.I. Shekhter, and M. Jonson, Phys. Rev. B 82, 125311 (2010).

19. D. Radi, A. Nordenfelt, A.M. Kadigrobov, R.I. Shekhter, M. Jonson, and L.Y. Gorelik, Phys. Rev. Lett. 107, 236802 (2011).

20. G.A. Skorobagat'ko, I.V. Krive, and R.I. Shekhter, Fiz. Nizk. Temp. 35, 1221 (2009) [Low Temp. Phys. 35, 949 (2009)]; I.V.
Krive, A. Paleuski, R.I. Shekhter, and M. Jonson, Fiz. Nizk. Temp. 36, 155 (2010) [Low Temp. Phys. 36, 119 (2010)].

21. L.Y. Gorelik, S.I. Kulinich, R.I. Shekhter, M. Jonson, and V.M. Vinokur, Phys. Rev. Lett. 95, 116806 (2005).

22. L.Y. Gorelik, S.I. Kulinich, R.I. Shekhter, M. Jonson, and V.M. Vinokur, Appl. Phys. Lett. 90, 192105 (2007). 